\title{
Mesenchymal stem cells regulated liver DX5- natural killer cells in acute-on-chronic liver failure
}

\section{Jing Xiong}

Department of infectious diseases, the third affiliated hospital of Sun Yet-Sen university, Guangzhou, China. GuangDong provincial key laboratory of liver diseases, the third affiliated hospital of Sun Yat-sen university, Guangzhou, China

\section{Weizhen Weng}

Department of infectious diseases, the third affiliated hospital of Sun Yet-Sen university, Guangzhou, China. Department of infectious diseases, the seventh Affiliated Hospital of Sun Yat-sen University, Shenzhen, China

\section{Dengna Lin}

Department of infectious diseases, the third affiliated hospital of Sun Yet-Sen university, Guangzhou, China. GuangDong provincial key laboratory of liver diseases, the third affiliated hospital of Sun Yat-sen university, Guangzhou, China

\section{Huijuan Cao}

Department of infectious diseases, the third affiliated hospital of Sun Yet-Sen university, Guangzhou, China

\section{Junfeng Chen}

Department of infectious diseases, the third affiliated hospital of Sun Yet-Sen university, Guangzhou, China

\section{Shaoquan Zhang}

Department of infectious diseases, the third affiliated hospital of Sun Yet-Sen university, Guangzhou, China

\section{Jing Zhang}

Department of infectious diseases, the third affiliated hospital of Sun Yet-Sen university, Guangzhou, China

\section{Juan Gao}

Department of infectious diseases, the third affiliated hospital of Sun Yet-Sen university, Guangzhou, China

\section{Ildiko Gyory}

Department of Molecular and Cell Biology, University of Leicester, UK

\section{Zhiliang Gao}

Department of infectious diseases, the third affiliated hospital of Sun Yet-Sen university, Guangzhou, China. GuangDong provincial key laboratory of liver diseases, the third affiliated hospital of Sun Yat-sen university, Guangzhou, China 


\section{Shiqiu Xiong}

Department of Molecular and Cell Biology, University of Leicester, UK

Bingliang Lin ( $\square$ linbingl@mail.sysu.edu.cn )

Third Affiliated Hospital of Sun Yat-Sen University

\section{Research}

Keywords: Acute-on-chronic liver failure; Bone marrow mesenchymal stem cell; Nature killer cell; GM-CSF.

Posted Date: August 1st, 2020

DOI: https://doi.org/10.21203/rs.3.rs-48005/v1

License: (c) (1) This work is licensed under a Creative Commons Attribution 4.0 International License.

Read Full License 


\section{Abstract}

Background :Acute-on-chronic liver failure (ACLF) is a severe, life-threatening syndrome with high mortality. Transplantation of Mesenchymal Stem Cells (MSCs) have been shown their efficacy in reducing mortality of ACLF patients in our clinical trials. Unveiling the underlying mechanism of action will improve clinical regime of ACLF.

Methods: Flow cytometry was applied to assess frequency and phenotype of peripheral blood natural killer cell (NK) from HBV-ACLF patients during MSCs treatment. An acute-on-chronic liver failure mouse model was established by injection of carbon tetrachloride (CCL4) intraperitoneally. We used flow cytometry to characterize frequency, phenotype, proliferation, cytokine secretion and cell conjugation of NK cells from different sources before and after MSCs treatment. Microscopy was applied to investigate cell-cell contact.

Results: Frequency of peripheral blood NK cells from HBV-ACLF patients was upregulated by MSCs infusion, which correlated with hepatitis remission. MSCs infusion rescued ACLF mice efficiently, coinciding with hepatitis remission, liver regeneration and NK cells frequency increase both in blood and liver. Strikingly, NKP46 ${ }^{+}$DX5, instead of NKP46 ${ }^{+}$DX5 $5^{+}$liver NK cells responded well to MSCs. In vitro, MSCs were proved to promote the proliferation and GM-CSF secretion of NKP46 ${ }^{+}$DX5 liver NK cells specifically, which may be owing to their high cell-cell adhesion.

Conclusions: Promoting NKP46 ${ }^{+}$DX5 ${ }^{-}$liver NK cells proliferation and secreting GM-CSF maybe the new machinery of MSCs' efficacy in ACLF.

\section{Background}

Acute-on-chronic liver failure (ACLF) was widely recognized as a distinct clinical entity featured with an acute deterioration of liver function in patients with chronic liver conditions. It was an unexpected rapid and abrupt decompensation of liver function with a high 28-days mortality ${ }^{[1,2]}$. In pathophysiology, systemic inflammation and susceptibility to opportunistic infection are typical features. Hepatic inflammation is closely related to hepatocytes injury and necrosis, which also contributes to systemic inflammation ${ }^{[3-5]}$. The general character of immune response in ACLF was similar to that of patients with severe sepsis, which caused hepatocyte apoptosis and necrosis. Both ACLF and severe sepsis showed a key feature of systemic inflammation, and their poor outcomes were closely associated with cytokine storm ${ }^{[6-8]}$. Currently, main therapeutic approaches for ACLF, included etiological treatment, artificial liver support systems, immune regulatory therapy, and liver transplantation. Among which, liver transplantation was the only definitely curable treatment, but its application was limited by the scarcity of organs donor, high costs, and life-long immunosuppression ${ }^{[9-11]}$.

Mesenchymal stem cell (MSCs) transplantation was a novel and promising cell therapy for ACLF ${ }^{[12,13]}$. The unique immune-regulatory and regenerative function of MSCs popularize them in the cellular 
treatment of autoimmunity and inflammation-mediated diseases, including graft versus host disease (GVHD), myocardial infarction, stroke, lupus, arthritis, Crohn's disease, acute lung injury, cirrhosis, multiple sclerosis and diabetes ${ }^{[13-15]}$. In our clinical trials, MSCs transplantation could improve the short-term survival rate of ACLF patients significantly ${ }^{[16]}$. The compelling decipher of the cellular and molecular machinery of MSCs transplantation in ACLF will optimize clinical therapeutic strategies and expand our understanding on pathophysiological basis.

Previous studies demonstrated that MSCs not only had strong multi-differentiation and self-renewal capabilities, but also played a vital role in immunomodulation ${ }^{[17,18]}$. Nature killer (NK) cells are the main group of innate immune cells,which consist the majority of non-parenchymal cells in liver. Up to $30-50 \%$ of human liver lymphocytes are NK cells, and their phenotype and functions are significantly different from the counterpart in spleen and peripheral blood ${ }^{[19]}$. Several in vitro studies indicated the interaction between human peripheral blood NK cells and MSCs; activated blood NK cells targeted and killed MSCs; meanwhile, MSCs inhibited activation of NK cell through down-regulating expression of the activating receptor and secretion of INF-y ${ }^{[20-22]}$. However, little is known about the interaction between MSCs and liver NK cell in vivo.

In this report, we observed that frequency and phenotype of peripheral blood NK cells from HBV-ACLF patients were significantly changed due to MSCs infusion, and the change of NK cells was correlated with hepatitis remission. With an adequate ACLF mouse model, MSCs transfusion significantly decreased morbidity of ACLF mice. MSCs treated ACLF mice showed less liver cell death and more liver regeneration. The frequency of liver and blood NK cell, instead of their counterpart from spleen, was enhanced subsequent to allogenic MSCs treatment. Most interestingly, the frequency of NKP46 ${ }^{+} D X 5^{-}$ liver NK, instead of their NKP46 ${ }^{+} D X 5^{+}$counterpart, was increased by MSCs transfusion. In vitro, these NKP46 ${ }^{+}$DX $5^{-}$NK could interact with MSCs, proliferated and secreted GM-CSF, which may be associated with liver regeneration and rescue efficacy following MSCs transfusion. To our knowledge, this is the first report suggested liver resident NK cells took part in MSC rescuing ACLF, which shed light on the novel role of these organ-specific NK cells.

\section{Methods}

\section{Clinical study and peripheral blood NK characterization}

The details in our clinical trial of allogenic MSCs treatment on HBV-ACLF patients has been reported ${ }^{[16]}$. Peripheral blood mononuclear cells (PBMC) were isolated from fresh peripheral blood of patients at week $0,2,4,8,12$ after MSCs treatment, and 13 healthy volunteers were recruited as well. $1 \times 10^{6} /$ sample was stained with Abs combinations. This study conformed strictly to the ethics guide- lines of the 1975 Declaration of Helsinki. The study protocol was approved by the ethics committee on clinical trials of the Third Affiliated Hospital of Sun Yat-sen University in 2010. 
For all multi-color flow cytometry, CS\&T beads (BD, 661414) were used for machine calibration and performance tracking. BD rainbow beads were for PMT voltage optimization (BD, 559123). Fluorescence minus one (FMO) was applied for positive cell gating. Anti-rat IgG negative control compensation particle set (BD, 552845) was used for fluorochrome compensation. All the samples were run on FACS LSRIl; and all data analyzed with FlowJo V10.

\section{Animals}

Four to six weeks Balb/c mice were applied our animal works in strict compliance with the policies and guidelines of Sun Yat-Sen University Institutional Animal Care and Use Committee. All mice were housed in specific pathogen-free conditions and exposed to a 12 hours daylight/darkness environment, and allowed unlimited access to food and water.

\section{$\mathrm{CCL}_{4}$ induced acute-on-chronic liver failure murine model}

Carbon tetrachloride (CCL4, Sigma, 319961) were diluted in olive oil(Sigma, 01514)into the concentration of $10 \%$ and $50 \%$ respectively. To induce liver cirrhosis, $10 \%$ CCL 4 was injected into Balb/c mice intraperitoneally by $5 \mathrm{ml} / \mathrm{kg}$ for 8 weeks continuously, twice a week. In day 3 of week 8 , a single sub-lethal dose of $\mathrm{CCL}_{4}(50 \%, 8 \mathrm{ml} / \mathrm{kg})$ was injected intraperitoneally to induce acute-on-chronic liver failure (Fig. 3A).

\section{ALT/AST test}

Mouse blood samples were collected from tail veins in $0,6,12,24$ and 48 hours after sub-lethal CCL4 challenged, centrifuged at $2000 \mathrm{rpm} / \mathrm{min}$ for $5 \mathrm{~min}$, the supernatants were collected. The levels of ALT and AST in the supernatants were detected according to instructions (Nanjing Jiancheng biological technologies).

\section{Liver H\&E staining}

After sub-lethal CCL4 challenged, experimental mice were sacrificed in $0,6,12,24$ and 48 hours along with mice from control groups. Mouse liver samples were fixed and embedded in paraffin. Sections were affixed to slides and de-paraffinized. Hematoxylin (Sigma, MHS16) and eosin (Sigma, 318906) were applied to stain cytoplasm and nucleus to define liver morphologic changes. The collagen fiber was stained with Sirius red (Sigma, 365548).

\section{Preparation of bone marrow mesenchymal stem cells}

Mouse MSCs were isolated from the femurs and tibiae of 4-6 week old male Balb/c mice according to modified methods reported previously ${ }^{[22,23]}$. Briefly, mouse bone marrow was collected from femurs and tibiae. After red blood cells lysis, cells were cultured in complete medium supplemented with $5 \mathrm{ng} / \mathrm{ml}$ fibroblast growth factor (bFGF, Peprotech, 450 - 33). 3 days later, suspension cells were washed away, 
$80 \%$ confluent adherent cells were trypsinized and passaged into new plate. After 3 rounds passage, $\geq$ $85 \%$ cells expressed MSCs marker. (Figure S1A,1B).

\section{Mononuclear cells purification from peripheral blood, liver and spleen}

Mice peripheral blood mononuclear cells (PBMC) were purified by OptiprepTM. Mouse spleen was minced, grinded, and filtered, then enriched by Ficoll. Mouse livers were dislodged after thorough perfusion with PBS. After being minced and grinded, liver suspension was digested in $0.05 \%$ collagenase IV (Sigma, C5138) and $0.001 \%$ DNasel (Sigma, 11284932001) in $37^{\circ} \mathrm{C}$ for $30 \mathrm{~min}$, then passed through 100-gauge stainless steel meshes and spun down. Liver mononuclear cells were purified by Percoll.

\section{Identify MSCs and characterize NK cells by flow cytometry}

After each passage, $0.5 \times 10^{6} \mathrm{MSCs}$-alike cells were harvested, washed, and re-suspended in $100 \mathrm{ul} \mathrm{FACS}$ buffer, stained with different Abs combinations or alone, along with the corresponding isotype control antibodies. Live/dead cell marker was included to exclude dead cells. To characterize human and mouse NK cells from peripheral blood, liver and spleen, different Abs combinations or alone were applied (Table S1).

\section{MSCs Transplantation}

ACLF mice were divided into two groups, 4-6 mice/group, ready for MSCs in saline or saline only transplantation. After 3 rounds of passage, MSCs were collected. After validation by surface marker, MSCs were re-suspended in sterile saline solution. 1 hour after sub-lethal dose of $\mathrm{CCL}_{4}(50 \%, 8 \mathrm{ml} / \mathrm{kg})$ intraperitoneal challenge, $0.5 \times 10^{6} \mathrm{MSCs}$ were infused via tail vein. Mice in control group were injected identical amount of saline.

\section{Hepatocellular death assay}

Cleaved caspase 3 was used as the readout of hepatocellular death by western blot. Briefly, mouse livers were treated and lysed in RIPA buffer. Samples were loaded and run in 10\% SDS-PAGE gel, then transferred overnight in cold room. Then membrane was blocked and incubated with 1:1000 diluted Caspase 3 antibody (Cell Signaling Technology) overnight in $4^{\circ} \mathrm{C}$. Then incubated with 1:5000 peroxidase-conjugated goat anti-rabbit IgG antibody (Asbio Technology, as006). $\beta$-actin was included as loading controls.

\section{Hepatocellular regeneration}

$75 \mathrm{mg} / \mathrm{kg}$ Bromodeoxyuridine (Brdu) (Sigma Aldrich) was injected into mice intraperitoneally after MSCs cell transplantation, another same dose Brdu pulsed in $0,1,6,12$, or 24 hours before sacrificing the mice. Paraffin-embedded liver tissue was cut into $5 \mu \mathrm{m}$ sections. Antigens were retrieval by microwave heating. After cool methanol fix, $5 \%$ goat serum was applied to block nonspecific binding. Following incubation 
with 1:250 anti-Brdu Ab (Abcam, Cambridge), then stained with secondary antibodies (Abcam, Cambridge). DAPI (Life Science) was also applied to stain nucleus.

\section{MSCs induced liver NK proliferation in vitro}

Peripheral blood NKP46 ${ }^{+} \mathrm{NK}$ cells and NKP46 ${ }^{+} \mathrm{DX} 5^{+/-}$liver NK cells were further purified via cell sorting on BD FACSAria II. Cells from 3-5 mice were pooled to get enough cell number. $0.5 \times 10^{6} /$ well NK cells were plated into $\mathrm{U}$ shape 24 well plate, $100 \mathrm{U} / \mathrm{ml}$ mouse IL-2 supplemented to maintain NK cells, plates were spun, and kept in incubator overnight, then labelled with cyto labeling red reagent (Abcam, ab176736)according to supplier's manual. These cells were cultured with or without MSC for 48 hours, then stained with DX5-PE-CF594 (BD, 562453) NKP46-Percp-Cy5.5 (BD, 560800), and live/dead cell fixable marker (Thermo Fisher, L34957), run samples on BD LSRII.

\section{MSCs induced liver NK activation in vitro}

NK cells were purified as above, then transferred into 24 well flat plate, with/without pre-coated with $80 \%$ confluent MSCs. After having cultured for 12 hours, $6 \mu \mathrm{g} / \mathrm{ml}$ Golgi-Stop (BD Biosciences, 554724) was added. In another 4 hours before suspension, the cells were harvested and stained with NKP46-PercpCy5.5 (BD, 560800), DX5-PE-CF594 (BD, 562453); CD3-APCcy7 (Biolegend, 100221), CD107a-brilliant violet 421 (biolegend, 328626), live/dead cell fixable marker (Thermo Fisher, L34957). Then cells were fixed, permeabilized and stained with IFN-r-APC (biolegend, 505810); GM-CSF-FITC (biolegend, 505404). Samples were analysed wtih BD LSRII.

\section{Cell conjugation assays}

Liver NK cells and MSCs were prepared as above, the cells were stained with PKH26 and PKH67 according to manuals. $50 \mathrm{uL}$ of $0.1 \times 10^{6} \mathrm{cell} / \mathrm{s} / \mathrm{ml}$ of NK cell and MSC cell were mixed in a 96 well Vbottom plate, briefly spun down, and incubated for 30 minutes. Conjugation was stopped by gently adding ice-cold $1 \%$ paraformaldehyde, and run sample via flow cytometry.

To image live cells contact, both fluorescent dye stained cells were placed in 8-well glass bottom chamber slides (Labtek) at $37^{\circ} \mathrm{C} / 5 \% \mathrm{CO}_{2}$, spun in $200 \mathrm{rpm}$ for 1 minute, and observed with an inverted laserscanning confocal fluorescence microscope (SP5, Leica) equipped with a $63 \times 1.2$ NA water immersion objective, and a resonance scanner. Laser wavelengths of 488 and $555 \mathrm{~nm}$ were used for the excitation of PKH67 and PKH26, respectively. Data was analyzed with Image J.

\section{Statistical Analysis}

All data were analyzed using Windows software SPSS version 13.0. Data of clinical and biochemical features were expressed as frequency and mean \pm standard deviation. The frequency was compared using Chi-square, and the quantitative data was compared using $t$ tests. Survival rates were calculated. The Kaplan-Meier curve was delineated, which was compared using Log-rank test. Spearman's correlation was used to confirm the relationship between two variables. Greenhouse-Geisser correction was performed to assess the variance of difference across multiple variables. One-way ANOVA analysis was 
for the significant comparison among three or more groups of quantitative data. P-values $<0.05$ were considered statistically significant.

\section{Results}

\section{MSC treatment reversed NK frequency in HBV-ACLF patients' peripheral blood, which was correlated with the recovery of liver function.}

To investigate the potential interaction between MSCs and NK cells, frequency and phenotype of peripheral blood NK cells were observed along with clinical implications in 21 HBV-ACLF patients receiving MSCs treatment. Among them, 3 patients deceased within 4 weeks, their NK frequency was lower than that from survival patients, and MSC didn't increase them (Fig. 1b). On the contrary, MSCs treatment significantly reversed the decreased NK cells frequency in peripheral blood from survived patients in 8 weeks and hence on (Fig. 1a), and the liver function also improved (supplementary Table 2). Interestingly, the liver function improvement was later than peripheral NK frequency increase (Fig. 1a and c). This observation implied NK\% increase might be a cause of liver recovery, instead of consequence. Phenotypically, frequency of NKG2A and KIR3DL1 on these peripheral blood NK cells was upregulated by MSCs treatment (Fig. 2a and b). On the contrary, frequency of NKG2D, Perforin were suppressed while FasL was increased under ACLF conditions. The MSCs treatment didn't reverse their level to normal (Fig. 2c-e); instead, these functional markers of NK cells showed strong correlation with liver function (supplementary Table 3). The phenotypic and functional characters are the general feature of newly proliferated NK cells.

\section{Establishment of ACLF murine model}

In order to investigate the role of MSCs and NK cells in rescuing ACLF patients, we established a stable and suitable ACLF animal model. Balb/c mice were administrated $10 \% \mathrm{CCL}_{4}$ for 8 weeks, and then a sublethal dose of $\mathrm{CCL}_{4}$ challenge ( $8 \mathrm{ml} / \mathrm{kg}$ ) induced classic massive hepatic necrosis (Fig. 3a and d). Seven of ten mice died within 48 hours after drug challenge as compared with control group (Fig. 3b). The ALT was increased from $1831 \pm 738 \mathrm{U} / \mathrm{L}$ to $36418 \pm 4755 \mathrm{U} / \mathrm{L}$, and the AST was enhanced from $1129 \pm$ $406 \mathrm{U} / \mathrm{L}$ to31397 $\pm 3986 \mathrm{U} / \mathrm{L}$ in 24 hours in the ACLF model (Fig. 3c and supplementary Fig. 2). Accordingly, the histochemical staining showed massive hepatic necrosis and hemorrhage with pseudo lobule background (Fig. 3d). This stable and reproducible strategy confirmed the establishment of a reliable ACLF mice model.

\section{Transplanted MSCs rescued ACLF mice}

To validate the efficacy of the MSCs on ACLF mice, MSCs were developed and verified in vitro (supplementary Fig. 1). Compared with 14 of 20 mice died within 48 hours from saline injected control 
groups, only 6 out of 20 mice died in MSCs transplantation group. There was a statistically difference of survival rate in Kaplan-Meier curve (Log-rank $\left.X^{2}=7.36, P=0.0067\right)$ (Fig. 4a).

Consistent with survival rate change, hepatic necrosis in the lobules was visually mitigated by MSCs administration, starting from 6 hours' transplantation. The portal/periportal inflammation was also reduced in experimental group (Fig. 4b). As expected, MSCs administration decreased ALT significantly at 6 hours, 12 hours and 24 hours after onset of ACLF (Fig. 4c). Furthermore, after MSCs treatment for 6 hours, bulk protein from liver of ACLF mice contained less cleaved caspase 3 , which indicated that apoptosis of liver cells induced by $\mathrm{CCL}_{4}$ was partially relieved by MSCs (Fig. 4d). All these data suggested MSCs administration rescued ACLF mice.

The regeneration ability for the liver is central to maintain hepatic homeostasis, it is also a common index to evaluate clinical efficacy subsequent to medical treatment. Brdu incorporation assay was applied to assess hepatocytes regeneration. In saline control group, some background labeling appeared. However, Brdu-labelled cells number was significantly increased after MSCs were transplanted for 12 and 24 hours (Fig. 4e and f), which indicated liver regeneration induced after MSCs infusion, as expected.

\subsection{MSCs increased frequency of liver $\mathrm{DX} 5^{-} \mathrm{NK}$ cells in vivo}

Echoed to the ACLF patients, the frequency of peripheral blood NK cells decreased in the ACLF mice, and liver NK cells frequency were higher than that of control mice, but there was no frequency change in spleen NK cells. This could be interpreted that peripheral blood NK cells chemotactically migrated into inflammatory liver. After MSC transfusion, both NK cell populations from peripheral blood and liver were significantly increased, but frequency of spleen NK cell was kept intact. These change indicated MSCs induced additional effect on NK cells more than chemotactic migration (Fig. 5a-c).

Further characterization of these NK cell change showed that MSC increased DX5 ${ }^{-}$instead of DX5 liver NK cells frequency. This change was accompanied with NKG2A\% increase in DX5- liver NK cells, reaching the level of NKG2A\% in DX5 $5^{+}$liver NK cells, which showed no change by MSC transfusion (Fig. $5 d-f$ ). Interestingly, the essential phenotype difference between $\mathrm{DX} 5^{-}$and $\mathrm{DX} 5^{+}$liver NK cells is NKG2A frequency, in which DX5 $5^{-}$liver NK cells express much lower frequency of NKG2A than DX5 $5^{+}$counterparts (supplementary Fig. 3). Apart from NKG2A\%, the MSCs transfusion increased Ly49A and Ly49C frequency in liver NK cells, and decreased NKG2D\%, but there was no significant difference between $D \times 5^{-}$ and DX5 $5^{+}$liver NK cells (supplementary Fig. 4).

\section{MSCs enhanced DX5- liver NK cells proliferation and secretion of GM-CSF in vitro}

To decipher the machinery of the effect of MSCs on liver NK cells, purified peripheral blood and liver NK cells from mice were co-cultured with MSCs in vitro. NKP46 ${ }^{+} \mathrm{DX} 5^{-}$liver cells, instead of blood NKP46 ${ }^{+}$ and $\mathrm{DX} 5^{+}$liver NK, showed cell proliferation subsequent to MSCs treatment, and this only happened in 
ACLF mice treated with MSCs group. Furthermore, after co-cultured with MSC, DX5- liver NK cells from ACLF mice could produce GM-CSF, whereas no change in DX5 ${ }^{+}$liver NK cells (Fig. 6a-b). Interestingly, MSCs altered IFN-gamma secretion from neither DX5 $5^{+}$nor DX5 $5^{-}$liver NK cells of normal mice (data didn't show). This GM-CSF secretion from proliferating DX5 ${ }^{-}$induced by MSCs improved the hepatocytes proliferation and regulated immunity, this may be one mechanism of MSCs' clinical efficacy on ACLF patients.

\section{DX5- liver NK cells showed strong adhesion with MSCs than DX5 ${ }^{+}$liver NK cells}

To understand the differential responses between DX5 $5^{+}$and DX5- liver NK cells imposed by MSCs

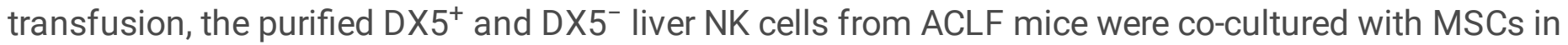
vitro, The DX5 ${ }^{-}$instead of DX5 ${ }^{+}$liver NK cells formed cell aggregates with MSCs (Fig. 7a-b). Besides NK cells proliferation, this also implied $\mathrm{DX} 5^{-}$cells may have stronger adhesion with MSCs than $\mathrm{DX} 5^{+}$cells, and this assumption was verified by cell-cell conjugation assay in vitro (Fig. 7c-d). This was in line with observed immune synapse between MSCs and liver DX5 ${ }^{-}$NK cells by live imaging (Fig. 7e), but we didn't manage to repeat this immune synapse formation on blood NK cells and DX5 $5^{+}$liver NK cells (data not shown). This stronger cell adhesion between MSCs and liver DX5- NK cells may attribute to the difference between liver DX5 $5^{-}$and $\mathrm{DX} 5^{+} \mathrm{NK}$ cells.

\section{Discussion}

Acute-on-chronic liver failure (ACLF) is defined as an acute deterioration in liver function on the basis of pre-existing chronic liver disease with high 28-d mortality. The pathogenic characters of ACLF are related to cytokine storm that induces systematic inflammatory response and subsequent immune paresis ${ }^{[4,24]}$. Currently, liver transplantation remains the definitive treatment with a good outcome ${ }^{[2]}$. However, Rapid disease progression and limited donor supply is driving scientists to explore novel treatment strategies ${ }^{[9]}$, including bone marrow-derived mesenchymal stem cells (MSCs) transplantation, which have achieved partial success in ACLF clinical treatment ${ }^{[25]}$.

MSCs are non-hematopoietic progenitor cells with potential to differentiate into multiple lineages of the mesenchyme. MSCs show distinguishing features on immune-privileged, immunomodulatory and tissue regeneration capacities, which highlighted them into one of the most promising therapies for ACLF $[13,15$, $18,21]$. Our recent clinical trials showed that infusion of allogeneic bone marrow-derived MSCs was safe and convenient for patients with HBV-related ACLF, and significantly increased the 24-week survival rate via an improvement of liver function and decreasing the incidence of severe infections ${ }^{[16]}$. But the underlying molecular and cellular machinery is unclear. Limited by availability of human liver tissue and suitable animal model, the regulation of MSCs on liver immune cells is rarely reported, although MSCs 
have showed to mainly migrate and reside in liver ${ }^{[26]}$. Therefore, we tried to probe the crosstalk between MSCs and liver immune cells in ACLF settings.

Our research revealed MSCs could increase the frequency of peripheral blood NK cells of patients with HBV associated ACLF, and the liver function improved synchronously, the same results were shown in mouse models. In ACLF mice, we found MSCs improved liver DX5 ${ }^{-}$NKP46 cells proliferation, GM-CSF secreting, and these two cells showed stronger cell-cell adhesion.

NK cells are the main subpopulation of leukocytes in the liver, and their physiological function are still largely unknown. Conventional NK cells from peripheral blood, spleen or lymph nodes are recognized as a subset of innate cytotoxic large granular lymphocyte, which recognize and eliminate stressed tumor or virus-infected cells ${ }^{[27]}$. Recent progresses suggest that liver NK cells are heterogeneous, consists of circulating and local resident populations which have different frequency, phenotype, development pathway, gene and protein expression profile and functions characters ${ }^{[28]}$. Liver resident NK cells preferentially performed immunological regulation, while circulating NK cells execute effector functions $[19,29]$. Previous reports confirmed cross-talk between human MSCs and NK cells in vitro ${ }^{[20,21,30]}$. These works guided us to assess the effect of MSCs on NK cells under ACLF context.

Limited by practical infeasibility of liver samples from ACLF patients, we only managed to look for surrogated peripheral blood NK cells changed by MSC infusion into HBV-ACLF patients. Consistent with previous reports, frequency of peripheral blood NK cells from ACLF patients was significantly decreased as compared with that of healthy volunteers ${ }^{[22]}$. This is mostly due to re-distribution of systemic leukocytes under several and acute inflammation in liver. After MSCs transfusion, the decrease of blood NK frequency was reversed, which nearly reached to healthy volunteers' level in week8. This peripheral NK frequency change was earlier than inflammation remission, such as INR decreased significantly in week 12. This phenomenon suggested that NK change maybe a cause of inflammation remission, instead of a consequence, if there is some direct relationship between them. Furthermore, patients with less peripheral $\mathrm{NK} \%$ had early and worse clinical outcome than those with higher NK\%, which also echoed our assumption.

To get some clue of the relationship between blood NK frequency change and inflammation emission, typical NK phenotypic markers were assessed. KIR3DL1 frequency on blood NK cells was increased instantly by MSCs transplantation, NKG2A frequency showed slight upregulation in late stage, and all others (KIR2DL1/2/3, NKG2D, FasL, Perforin, Granzyme B) kept intact. To our knowledge, this unique profile of NK receptors change hasn't been reported before, which implied that MSCs had unique impact on NK cells from ACLF patients.

To understand the cellular and molecular machinery of MSCs on liver NK cells in ACLF setting, we elaborately established chronic liver damage mouse model by intraperitoneal injection of low-dose CCL4 for eight weeks to induce cirrhosis. The ACLF occurs in the setting of a progressive chronic systemic inflammation, mostly liver cirrhosis ${ }^{[6]}$. To initiate an acute burst of liver damage and systemic 
inflammation, a sub-lethal dose of CCL4 was injected. Our data showed that the mouse death rate reached $70 \%$ within 6 hours after challenge, which is comparable to the high mortality of ACLF patients. In line with this, the liver histological structure was deformed and serum aminotransferase level was strikingly elevated, which strongly implied that CCL4 induced liver damage was responsible for the mouse death. Furthermore, more caspase 3 was cleaved after challenge indicated apoptosis involved in CCL4 mediated liver damage, which excluded the complete hepatic toxicity induced by CCL4 in this model. Significant lymphocytes infiltration in the liver after CCL4 challenge indicated the exacerbated systemic inflammatory responses, which matched the paramount pathogenic characters of human ACLF. According to widely-acknowledged criteria for ACLF mouse model ${ }^{[31,32]}$, our optimized experimental conditions produced adequate ACLF murine model.

Owing to tissue scarceness of primary MSCs, it is necessary to proliferate and expand them through cell culture. Their fate and function in culture is influenced by various external factors, including the specific cell source, donor age, plating density, passage number, plastic surface quality and external cytokines ${ }^{[33]}$. After long-term comparison and optimization, the MSCs under our culture conditions showed typical phenotype and functional recovery of ACLF mice. In line with the decreased death rate, autogenic MSCs alleviated liver damage and promoted hepatic regeneration in ACLF mice.

As expected, the expanded MSCs significantly increased survival rate of ACLF mice, which was consistent with other reports ${ }^{[12,15,34,35]}$ and our clinical trials ${ }^{[16]}$. The mechanism may attribute to MSCs' capacity of immune regulation and regeneration ${ }^{[14]}$. Although it was found that MSCs could promote cell proliferation in liver, it is hard to tell these were hepatocytes or liver immune cells. However, there was significant inflammatory remission and less cell death after MSCs transplantation, which emphasized their role in immune regulation, and echoed our observation in HBV-ACLF patients. Hence, the NK cells were specifically investigated w/o MSCs in ACLF settings.

Similar to HBV-ACLF patients, MSCs infusion upregulated frequency of circulating peripheral blood NK cells in ACLF mice, which also showed in liver instead of spleen. Both liver and peripheral blood contain higher frequency of NK cells, but spleen keep intact after MSCs transfusion. It is more likely that there were more NK proliferation and development, instead of immune cells redistribution only after liver inflammatory remission.

More and more evidence proved that liver NK cells could be stratified into conventional circulating NK cells (NKP46 ${ }^{+} D X 5^{+}$) and local resident liver NK cells (NKP46 $\left.{ }^{+} D X 5^{-}\right)$. DX5 (or CD49b) has been recently characterized by $150 \mathrm{KD}$ integrin, which non-covalently associates with CD29 ( $\beta 1$ integrin) to form the CD49b/CD29 complex regulating NK or T cells. A lot of proof showed that these two subpopulations differ in their development stage, phenotypic markers, transcription factor profile and functional characters ${ }^{[28]}$. Reports showed liver NKP46 ${ }^{+} \mathrm{DX} 5^{-}$cells involved in the pathogenesis of different liver disease ${ }^{[36-38]}$, and they proliferated under specific circumstance ${ }^{[39]}$.Strikingly, frequency of NKP46 ${ }^{+} \mathrm{DX} 5^{-}$ cells upregulated by MSCs infusion along with higher NKG2A expression. Except for possible liver NKP $46^{+} \mathrm{DX} 5^{+}$cells re-joined blood circulation after liver inflammatory remission, it was likely that new NK 
produced considering the change of NKG2A frequency. In vitro co-culture of purified NK and MSCs varified this assumption, the MSCs promoted proliferation of NKP46 $D \times 5^{-}$liver cells, instead of NKP $46^{+} D X 5^{+}$liver cells or blood NK cells. Furthermore, more GM-CSF was produced from NKP $46^{+} D X 5^{-}$ liver cells by MSCs.

Granulocyte-macrophage colony-stimulating factor (GM-CSF) was originally classified as a

hematopoietic growth factor ${ }^{[40]}$. Recent rat model showed GM-CSF involved in biliary wound healing and hepatocellular regeneration ${ }^{[40,41]}$. GM-CSF has also been used in the treatment of sepsis-associated immunosuppression, enhancement of lymphoid and myeloid subpopulations ${ }^{[42]}$. Considering the similar immunological characters between sepsis and ACLF, it is reasonable to propose that GM-CSF restored immune competency and/or enhance liver regeneration in ACLF patients.

To further investigate the potential machinery of MSCs' preference on $\mathrm{DX} 5^{-}$instead of $\mathrm{DX} 5^{+}$cells, purified NK cells were co-cultured with MSCs in vitro. The liver DX5 $5^{-}$NK cells showed higher cell-cell conjugation potency with MSCs than DX5 ${ }^{+} \mathrm{NK}$ cells, which may be associated with significant proliferation of liver NK cells induced by MSCs. Previous reports showed NK cells killed MSCs in vitro, we didn't get this ${ }^{[43]}$.The reason may be due to the different experimental model and conditions.

\section{Conclusions}

In this study, we showed MSCs infusion reversed frequency of peripheral blood NK in HBV-ACLF patients, accompanied with hepatic inflammation recession. Employed by the adequate and stable CCL4 induced ACLF mice model, the transfusion of MSCs rescued mice from ACLF-caused death. The potential mechanism involved the change of liver and blood NK frequency and phenotype. The present study demonstrated that the MSCs could interact with DX5- NK cells, which implied that there was a novel machinery of MSCs to rescue ACLF patients.

\section{Abbreviations}

ACLF:acute-on-chronic liver failure; MSC: mesenchymal stem cell; NK cell: natural killer cell; CCL4: carbon tetrachloride; GVHD: graft versus host disease; PBMC: Peripheral blood mononuclear cells; Brdu: bromodeoxyuridine; GM-CSF: granulocyte-macrophage colony-stimulating factor.

\section{Declarations}

\section{Ethics approval and consent to participate}

This study conformed strictly to the ethics guide- lines of the 1975 Declaration of Helsinki. The study protocol was approved by the ethics committee on clinical trials of the Third Affiliated Hospital of Sun Yat-sen University in 2010. Experiments on animals were carried out in compliance with the policies and guidelines of Sun Yat-Sen University Institutional Animal Care and Use Committee. 


\section{Consent for publication}

Not applicable

\section{Availability of data and materials}

All data generated or analysed during this study are included in this published article and its supplementary information files.

\section{Competing interests}

The authors declare that they have no competing interests.

\section{Funding}

This research was supported by National Science and Technology Major Project (2017ZX 10203201003, 2016ZX10002008, 2016ZX10002009, 2016ZX10002010) and Guangzhou Major Project in collaborative innovation of industry (201508020117).

\section{Authors' Contributions $\rrbracket$}

Bingliang Lin, Jing xiong, Shiqiu Xiong and Zhiliang Gao contrived and designed the study; Bingliang Lin, Jing xiong, Dengna Lin, Shaoquan Zhang, Huijuan Cao, Weizhen Weng, Junfeng Chen, Jing Zhang, Juan Gao performed experiments and Data analysis. Bingliang Lin, Shiqiu Xiong, and Ildiko Gyory wrote manuscript. The manuscript has been seen and approved by all authors.

\section{Acknowledgments}

We thank Professor Peng Andy Xiang, Xiao-Yong Chen working in Center for Stem Cell Biology and Tissue Engineering, Sun Yat-Sen University. We thank Kewei Wang working in Haerbin Medical University for her help in proofreading this paper.

\section{References}

1. Gustot T, Fernandez J, Garcia E, Morando F, Caraceni P,Alessandria C,et al. Clinical Course of acuteon-chronic liver failure syndrome and effects on prognosis. Hepatology. 2015; 62:243-52.

2. Sarin SK, and Choudhury A. Acute-on-chronic liver failure: terminology, mechanisms and management. Nat Rev Gastroenterol Hepatol.2016;13: 131-49.

3. Jalan R, Gines P, Olson JC, Mookerjee RP, Moreau R, Garcia-Tsao G, et al. Acute -on chronic liver failure. J Hepatol 2012; 57: 1336-48.

4. Bernal W, Jalan R, Quaglia A, Simpson K, Wendon J, Burroughs A, et al. Acute- on-chronic liver failure.Lancet.2015; 386: 1576-87. 
5. Asrani SK, Simonetto DA and Kamath PS.Acute-on-Chronic Liver Failure. Clin Gastroenterol Hepatol.2015;13: 2128-39.

6. Arroyo V and Moreau R. Diagnosis and prognosis of acute on chronic liver failure (ACLF) in cirrhosis. J Hepatol.2017;66:451-453.

7. Claria J, Arroyo V and Moreau R.The Acute-on-Chronic Liver Failure Syndrome, or When the Innate Immune System Goes Astray. J Immunol. 2016;197: 3755-3761.

8. Wasmuth HE, Kunz D, Yagmur E, Timmer-Stranghöner A, Vidacek D, Siewert E, et al. Patients with acute on chronic liver failure display "sepsis-like" immune paralysis. J Hepatol.2005;42:195-201.

9. Finkenstedt A, Nachbaur K, Zoller H, Joannidis M, Pratschke J, Graziadei IW, et al. Acute-on-chronic liver failure: excellent outcomes after liver transplantation but high mortality on the wait list. Liver Transpl.2013;19: 879-86.

10. Liu CL, Fan ST, Lo CM, Wei WI, Yong BH, Lai CL, et al. Live-donor live transplantation for acute-onchronic hepatitis B liver failure.Transplantation. 2003;76: 1174-9.

11. Chan AC, Fan ST, Lo CM, Liu CL, Chan SC, Ng KK, et al. Liver transplantation for acute-on-chronic liver failure. Hepatol Int.2009;3: 571-581.

12. Volarevic V, Nurkovic J, Arsenijevic N, Stojkovic M. Concise review: Therapeutic potential of mesenchymal stem cells for the treatment of acute liver failure and cirrhosis. Stem Cells.2014;32: 2818-23.

13. Uccelli A, L Moretta, and V Pistoia. Mesenchymal stem cells in health and Nat Rev Immunol.2008;8:726-36.

14. Shi Y, Hu G, Su J, Li W, Chen Q, Shou P, et al.Mesenchymal stem cells: a new strategy for immunosuppression and tissue repair. Cell Res.2010; 20:510-8.

15. Caplan Al and Correa D.The MSC: an injury drugstore. Cell Stem Cell. 2011;9:11-5.

16. Lin BL, Chen JF, Qiu WH, Wang KW, Xie DY, Chen XY, etal. Allogeneic bone marrow-derived mesenchymal stromal cells for hepatitis $B$ virus-related acute-on-chronic liver failure: A randomized controlled trial. Hepatology. 2017;66: 209-219.

17. Peister A, Mellad JA, Larson BL, Hall BM, Gibson LF, Prockop DJ. Adult Stem cells from bone marrow (MSCs) isolated from different strains of inbred mice vary in surface epitopes, rates of proliferation, and differentiation potential. Blood.2004;103: 1662-8.

18. Nauta AJ and Fibbe WE. Immunomodulatory properties of mesenchymal stromal cells. Blood.2007;110:3499-506.

19. Shi FD, Ljunggren HG, La Cava A, Van Kaer L.Organ-specific features of natural killer cells. Nat Rev Immunol.2011;11: 658-71.

20. Sotiropoulou PA, Perez SA, Gritzapis AD, Baxevanis CN, Papamichail M. Interactions between human mesenchymal stem cells and natural killer cells. Stem Cells.2006;24:74-85.

21. Spaggiari GM, Capobianco A, Abdelrazik H, Becchetti F, Mingari MC, Moretta L. Mesenchymal stem cells inhibit natural killer-cell proliferation, cytotoxicity,and cytokine production:role of indoleamine 
2,3-dioxygenase and prostaglandin E2.Blood.2008;111: 1327-33.

22. Zou Y, Chen T, Han M, Wang H, Yan W, Song G, et al. Increased killing of liver NK cells by Fas/Fas ligand and NKG2D/NKG2D ligand contributes to hepatocyte necrosis in virus-induced liver failure. J Immunol.2010; $184:$ 466-75.

23. Lei J, Hui D, Huang W, Liao Y, Yang L, Liu L, et al.Heterogeneity of the biological properties and gene expression profiles of murine bone marrow stromal cells. Int J Biochem Cell Biol.2013;45: 2431-43.

24. Rahimi, RS and Rockey DC, Acute on chronic liver failure: definitions, treatments and outcomes. Curr Opin Gastroenterol.2016;32:172-81.

25. Bianco P, Cao X, Frenette PS, Mao JJ, Robey PG, Simmons PJ,et al.The meaning,the sense and the significance: translating the science of mesenchymalstem cells into medicine. Nat Med.2013;19: 3542.

26. Eggenhofer E, Benseler V, Kroemer A, Popp FC, Geissler EK, Schlitt HJ, et al. Mesenchymal stem cells are short-lived and do not migrate beyond the lungs after intravenous infusion.Front Immunol.2012;3:297.

27. Vivier E, Ugolini S, Blaise D, Chabannon C, Brossay L.Tissue-resident natural killer cells in the livers. Nat Rev Immunol.2012;12: 239-52.

28. Peng $\mathrm{H}$ and Tian Z.Tissue-resident natural killer cells in the livers. Sci China Life Sci.2016; 59:12181223.

29. Xiong S, Sharkey AM, Kennedy PR, Gardner L, Farrell LE, Chazara O, et al. Maternal uterine NK cellactivating receptor KIR2DS1 enhances placentation. J Clin Invest.2013;123:4264-72.

30. Consentius C, Akyüz L, Schmidt-Lucke JA, Tschöpe C, Pinzur L, Ofir R, et al.Mesenchymal Stromal Cells Prevent Allostimulation In Vivo and Control Checkpoints of Th1 Priming: Migration of Human DC to Lymph Nodes and NK Cell Activation. Stem Cells.2015;33:3087-99.

31. Bélanger $M$ and Butterworth RF. Acute liver failure: a critical appraisal of available animal models.Metab Brain Dis.2005;20:409-23.

32. Maes $M$, Vinken $M$ and Jaeschke $H$. Experimental models of Hepatotoxicity related to acute liver failure.Toxicol Appl Pharmacol.2016;290:86-97.

33. Sisakhtnezhad S, Alimoradi E and Akrami H. External factors influencing mesenchymal stem cell fate in vitro. Eur J Cell Biol.2017; 96:13-33.

34. Russo FP and Parola M. Stem and progenitor cells in liver regeneration and repair. Cytotherapy.2011;13:135-44.

35. Wang J, Liao L and Tan J. Mesenchymal-stem-cell-based experimental and clinical trials: current status and open questions. Expert Opin Biol Ther. 2011;11:893-909.

36. Peng $\mathrm{H}$ and Sun R. Liver-resident NK cells and their potential functions.Cell Mol Immunol.2017;14:890-94.

37. Saeki Y, Ishiyama K, Ishida N, Tanaka Y, Ohdan H. Memory-like Liver Natural Killer Cells are Responsible for Islet Destruction in Secondary Islet Transplantation. Sci Rep.2019;9:1022. 
38. Nakano R, Ohira M, Yano T, Imaoka Y, Tanaka Y, Ohdan H. Hepatic irradiation persistently eliminates liver resident NK cells. PLoS One.2018; 13:e0198904.

39. Martrus G, Kautz T, Lunemann S, Richert L, Glau L, Salzberger W, Wang CY, et al. Proliferative capacity exhibited by human liver-resident CD49a+CD25+ NK cells. PLoS One.2017;12:e0182532.

40. Becher B, Tugues S, and Greter M. GM-CSF:From Growth Factor to Central Mediator of Tissue Inflammation.Immunity.2016;45:963-973.

41. Meng F, Francis H, Glaser S, Han Y, DeMorrow S, Stokes A, et al. Role of stem cell factor and granulocyte colony-stimulating factor in remodeling during liver regeneration. Hepatology.2012;55:209-21.

42. Hutchins NA, Unsinger J, Hotchkiss RS, Ayala A. The new normal: immunomo- dulatory agents against sepsis immune suppression. Trends Mol Med. 2014;20:224-33.

43. Spaggiari GM, Capobianco A, Becchetti S, Mingari MC, Moretta L. Mesenchymal stem cell-natural killer cell interactions: evidence that activated NK cells are capable of killing MSCs, whereas MSCs can inhibit IL-2-induced NK-cell proliferation. Blood.2006;107:1484-90.

\section{Figures}



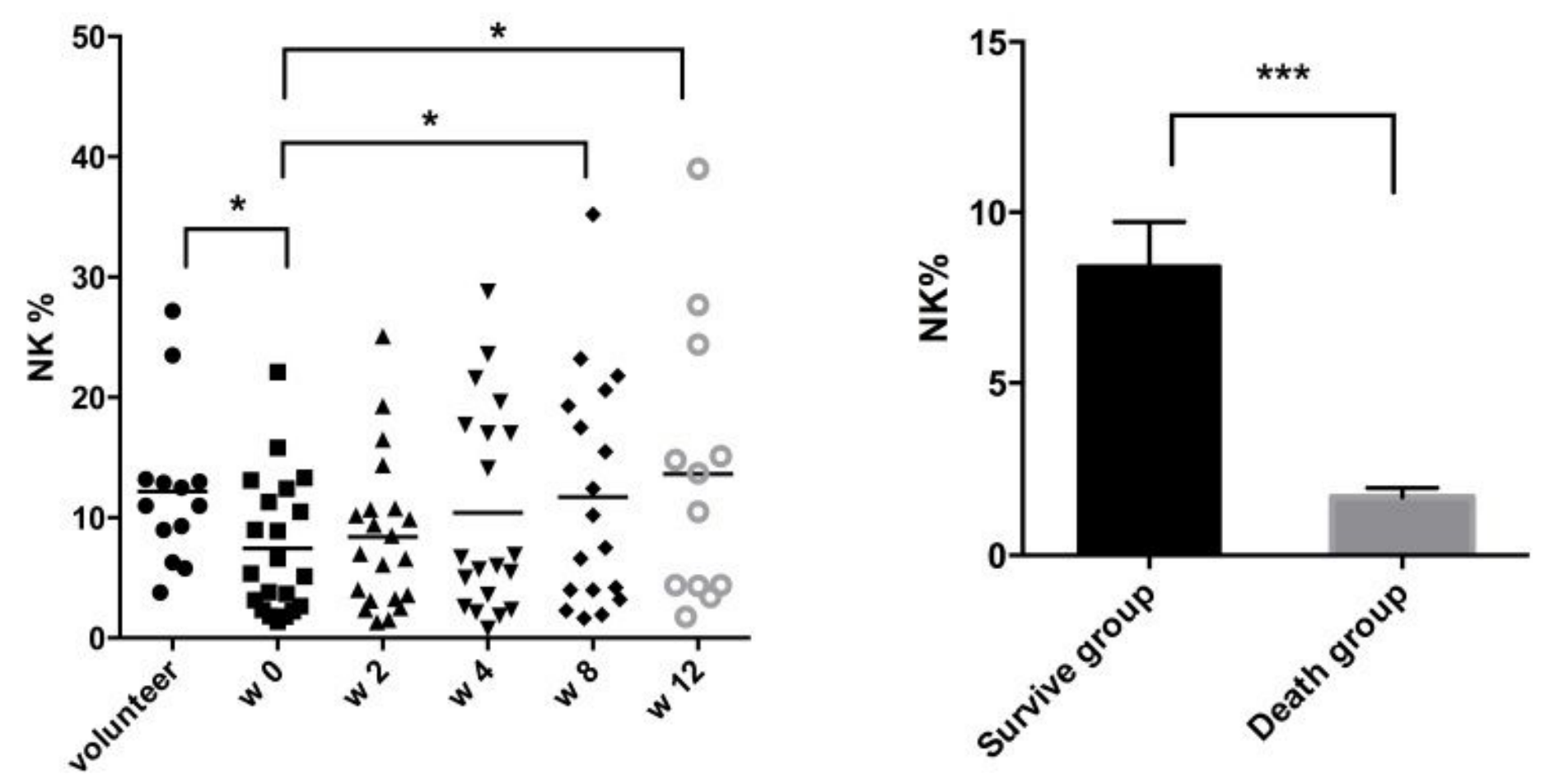

c

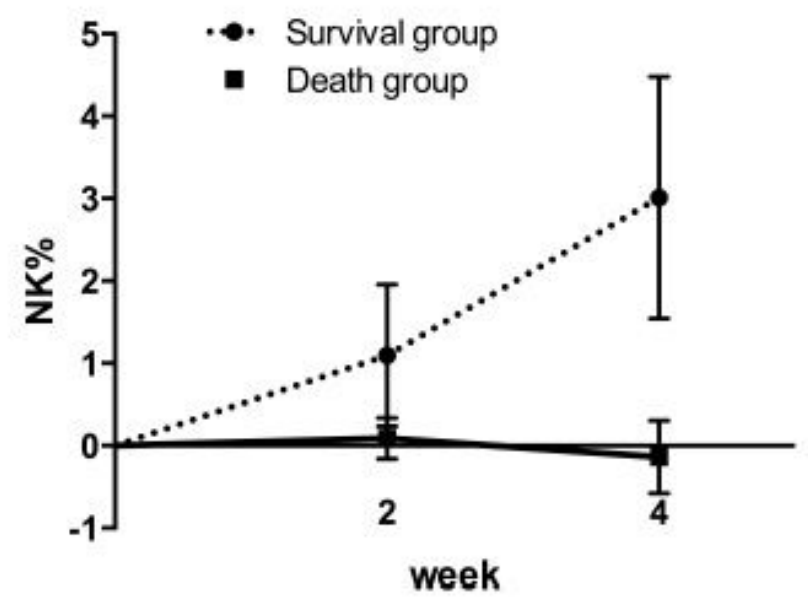

Figure 1

Frequency of NK cell in PBMC from HBV-ACLF patients was reversed by MSC treatment. (a) Frequency of CD3-CD56+ NK cells was assessed in PBMC from healthy volunteers $(n=13)$; HBV-ACLF patients treated with MSC on week $0(n=21)$; week $2(n=21)$; week $4(n=20)$; week $8(n=18)$ and week $12(n=12)$. (mean \pm s.e.m. ${ }^{*}<0.05$ by Paired-Samples t-Tests). (b) After MSC treatment and according to the final outcomes,

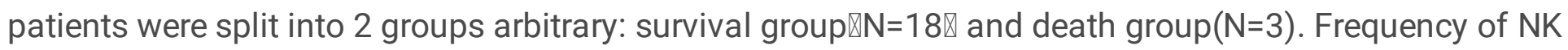
cells were higher in survival group, (mean \pm s.e.m. ${ }^{*} * \mathrm{P}<0.0001$ by Student's t-Tests). (c)The frequency of 
NK increased from the base line at week 2 and 4 between survival group $(\mathrm{N}=18)$ and Death group $(\mathrm{N}=3)$. (mean \pm s.e.m. ${ }^{*} \mathrm{P}>0.05$ by Student's t-Tests).

a

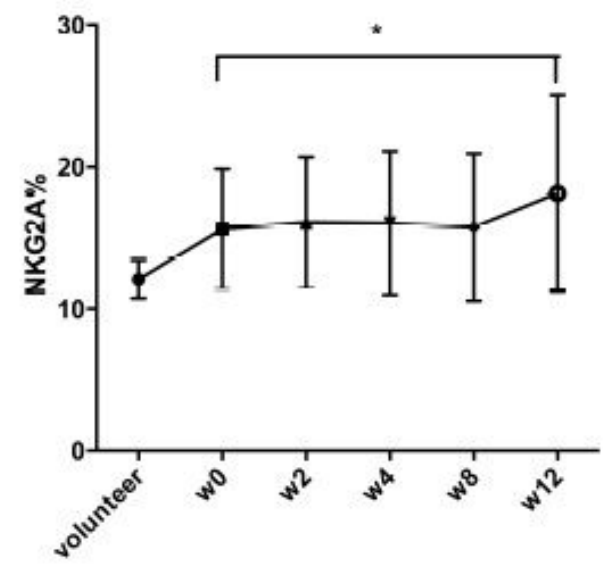

c

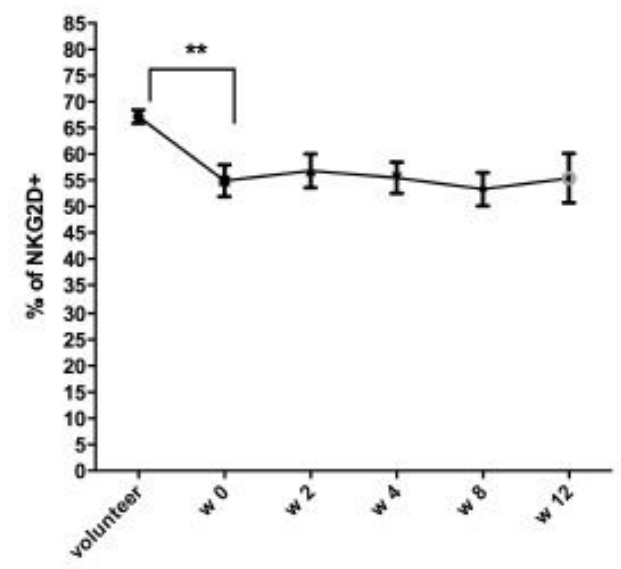

e

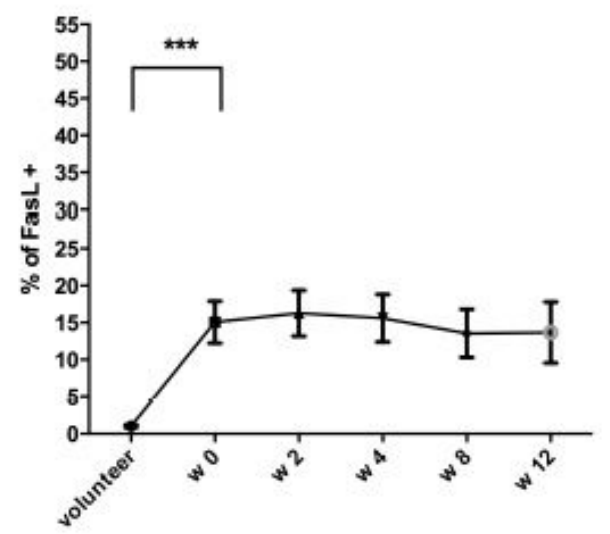

b

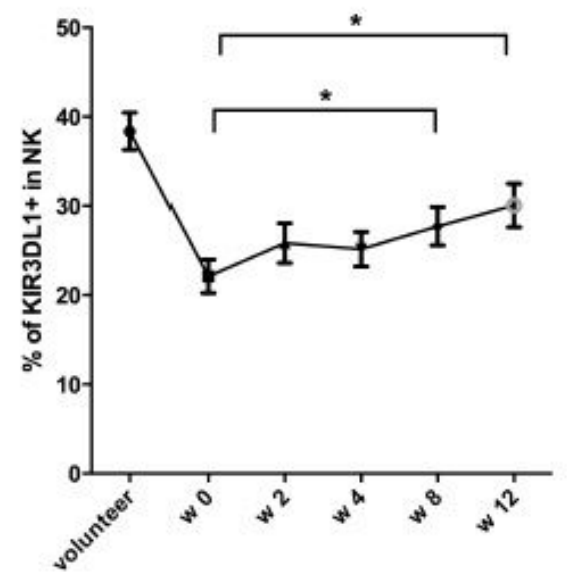

d

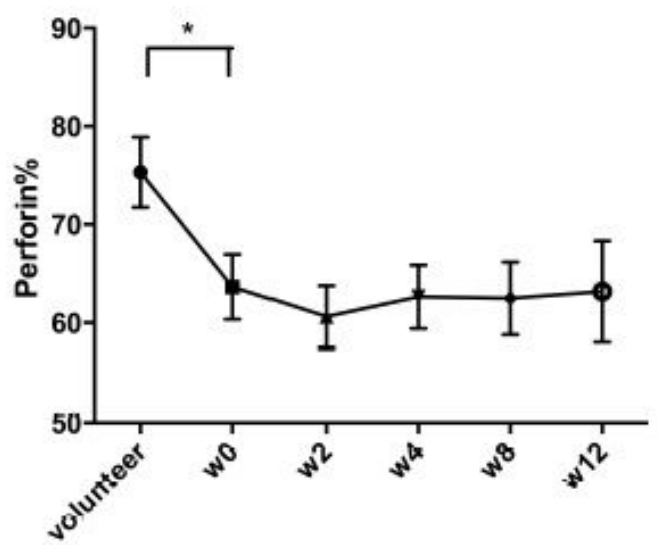

Figure 2

.MSC induced phenotypic change in NK cells from PBMC. a-e Frequency of NKG2A+ (a), KIR3DL1+ (b), NKG2D+ (c), Perforin+ (d) and FasL+ (e) cell in CD3-CD56+ NK cells from PBMC were assessed in healthy 
volunteer ( $n=13)$; HBV-ACLF treated with MSC on week $0(n=21)$; week $2(n=21)$; week $4(n=20)$; week $8(n=18)$ and week $12(n=12)$. (mean \pm s.e.m. ${ }^{*} P<0.05$ by Paired-Samples t-Tests).

a

b
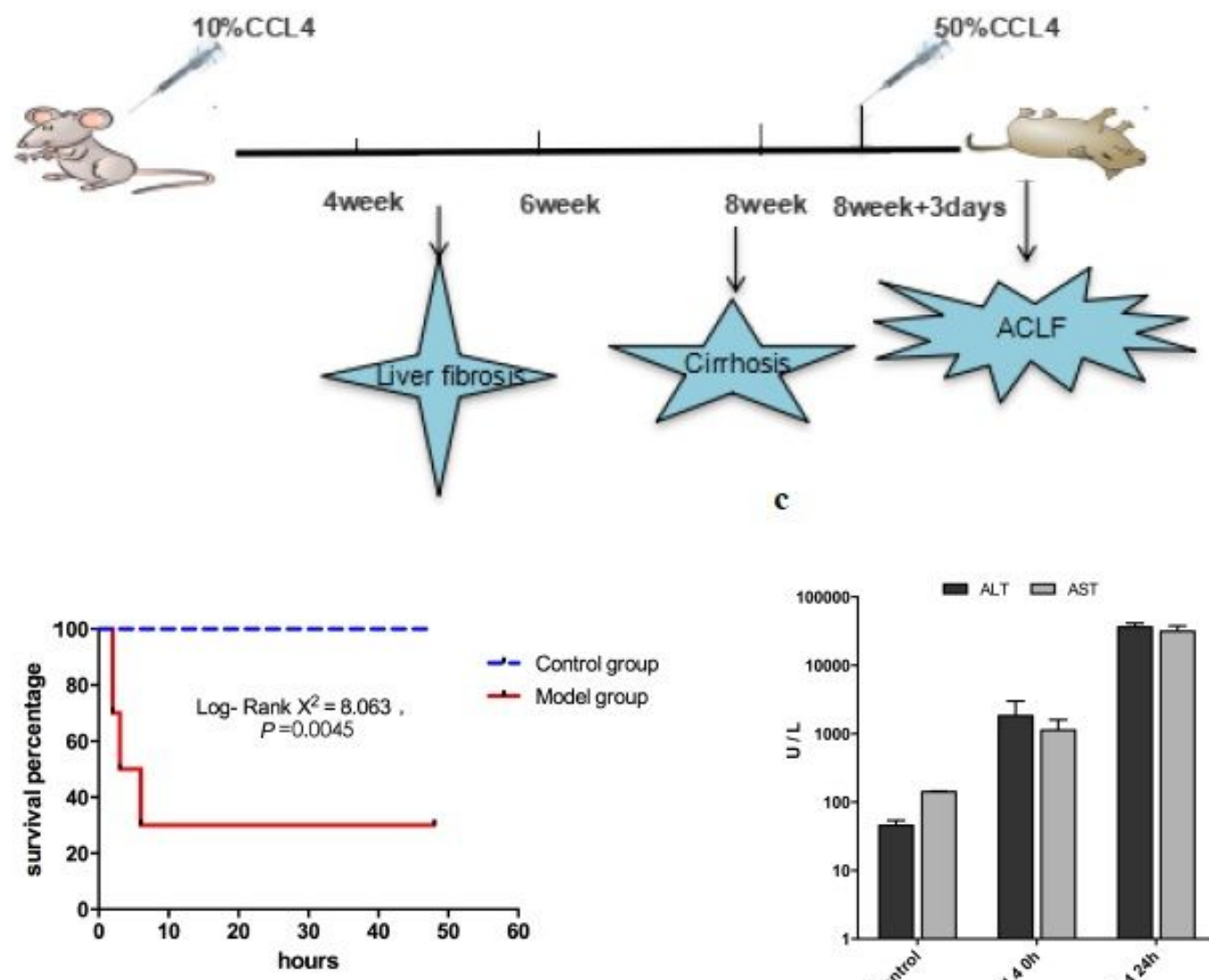

d

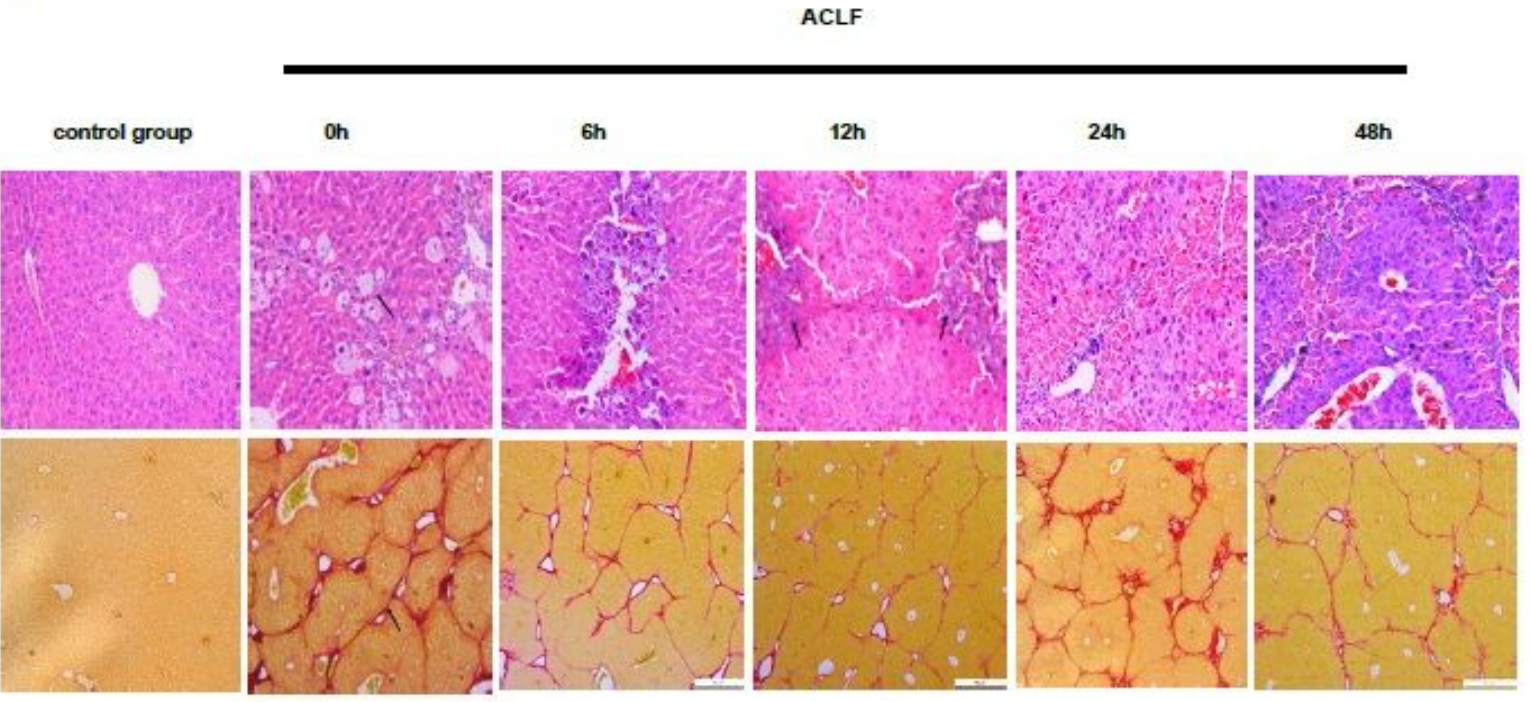

\section{Figure 3}

Establishment of ACLF animal model. (a) The schematic strategy of animal manipulation. (b) The survival curve of mice challenged by sub-lethal CCL4. Both groups consist of 10 mice. 3 mice died within 2h, 2 mice died at $3 \mathrm{~h}$ and 2 mice died at $6 \mathrm{~h}$ in ACLF model Group. This is a presentative result from 3 
experiments. (c) The serum transaminase levels in oil-only administered normal animal (Cont), 50\% CCL4 pulse transfusion for 0 hour (CCL4 Oh) and 24 hours (CCL4 $24 \mathrm{~h}$ ), $n=4$. The ALT/AST was increased from Oh to $12 \mathrm{~h}$ (mean \pm s.e.m , $\mathrm{P}=0.002 / \mathrm{P}=0.0016$ by Student's $\mathrm{t}$-Tests).(d) The upper row showed liver samples under $\mathrm{H} \& \mathrm{E}$ staining, arrow indicated filtrated lymphocytes, original magnification $200 \mathrm{x}$. The lower row indicated samples with Sirius Red staining, arrow indicates fibrosis, original magnification 50x. Similar results obtained from 3 individual experiments.

a

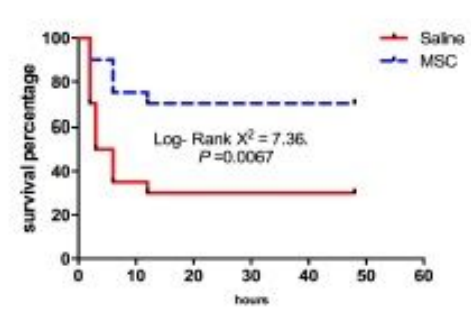

c

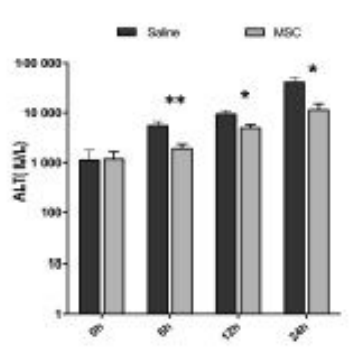

d
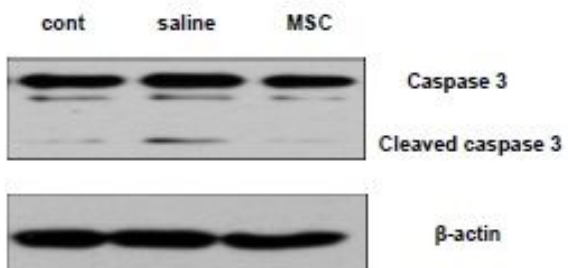

b

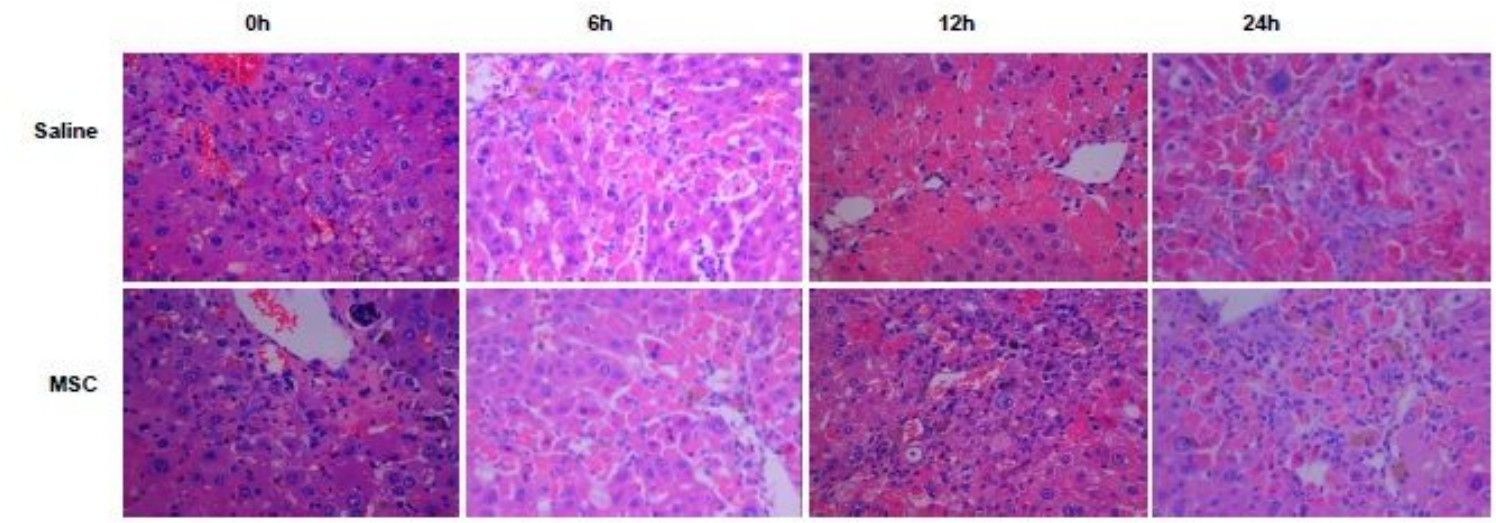

e

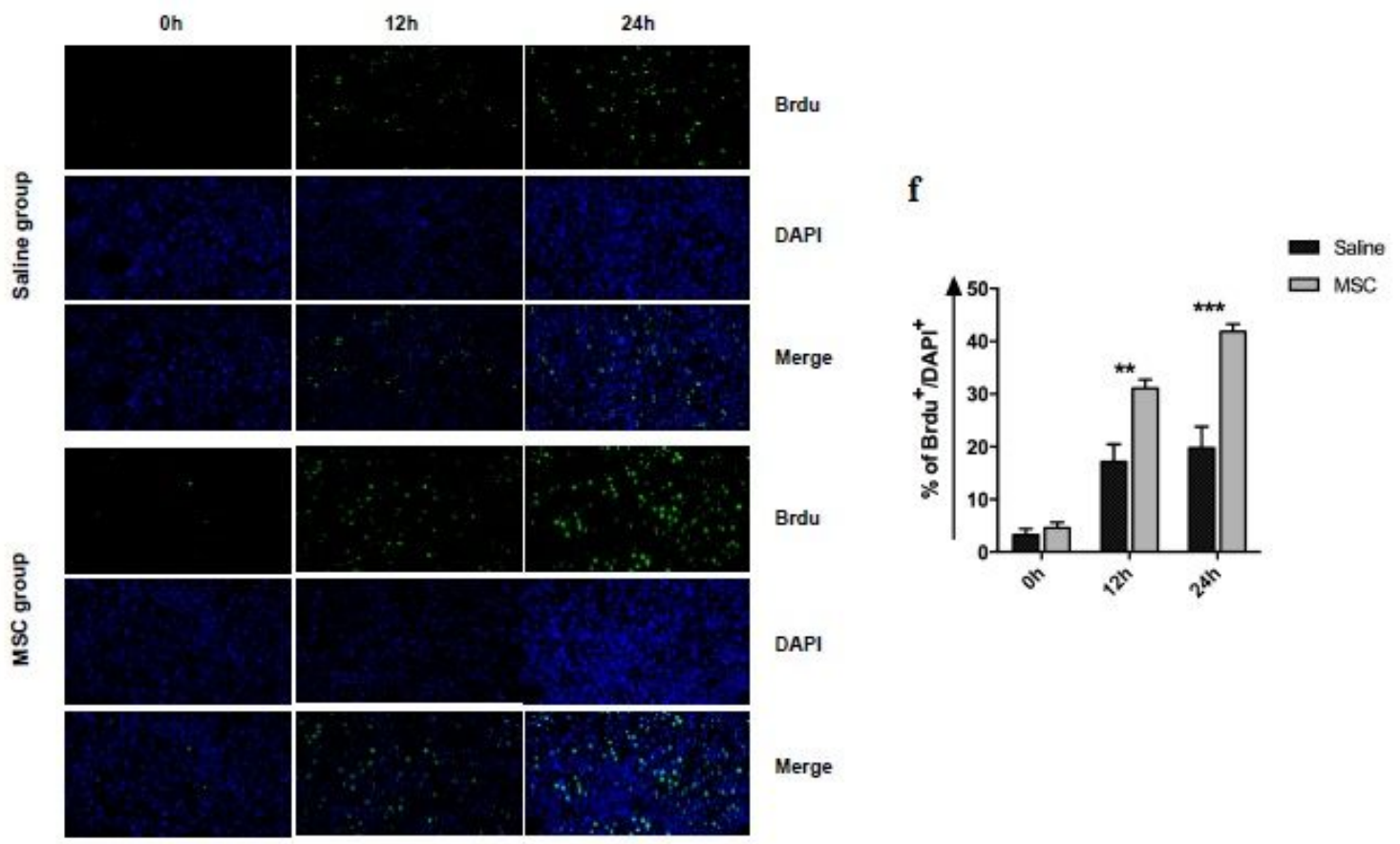

Figure 4 
Transfused MSCs partially rescued mice from ACLF-induced death, alleviated liver damage and enhanced liver proliferation. (a) The survival curve of CCL4 challenged ACLF mice with/without MSCs transplantation (saline/MSC group). Both groups consist of 10 mice. 6 mice died within $2 \mathrm{~h}, 4$ mice died at $3 \mathrm{~h}, 3$ mice died at $6 \mathrm{~h}$, and 1 mice died at $12 \mathrm{~h}$ in saline treatment group. In comparison $\otimes 2$ mice died within $2 \mathrm{~h} \otimes 3$ mice died at $6 \mathrm{~h}$ and 1 mice died at $12 \mathrm{~h}$ in MSC treatment Group. (b) H\&E stained liver samples from ACLF mice with/without MSCs transplantation (Saline/MSC group) for different time. 10x magnification. (c) The serum transaminase levels from mice with/without MSCs administration (saline and MSC treated group) in different time points. After MSC transplantation, ALT decreased from $5468 \pm$ 673 to $1895 \pm 384$ at $6 \mathrm{~h}(\mathrm{p}=0.0099)$, decreased form $9317 \pm 1225$ to $4897 \pm 461$ at $12 \mathrm{~h}(\mathrm{p}=0.0279)$ and decreased from $40745 \pm 6054$ to $11345 \pm 2070$ at $24 \mathrm{~h}(\mathrm{p}=0.01)$. Student T-test. $\mathrm{n}=3$, * * indicate $\mathrm{P}<0.01$, * indicate $P<0.05$. (d) Cleaved and total caspase 3 from mice liver with/without MSCs transplantation (saline/MSC group) after MSCs transfusion for 12 hours. Cont represented normal control. $\beta$-actin controlled loading. Similar results obtained from 3 individual experiments. (e) Brdu labelled mice liver from ACLF mice w/o MSCs transplantation (Saline/ MSC transfusion group) on different time points. Nuclei was highlighted by DAPI, Brdu labelled in green. Similar results obtained from 3 individual experiments. (f) Quantification of Brdu labelled cells by Image J. Student T test, $n=3$, ** indicate $P<0.01$, ${ }^{\star \star \star}$ indicate $P<0.001$ between saline and MSC treated groups. 
a

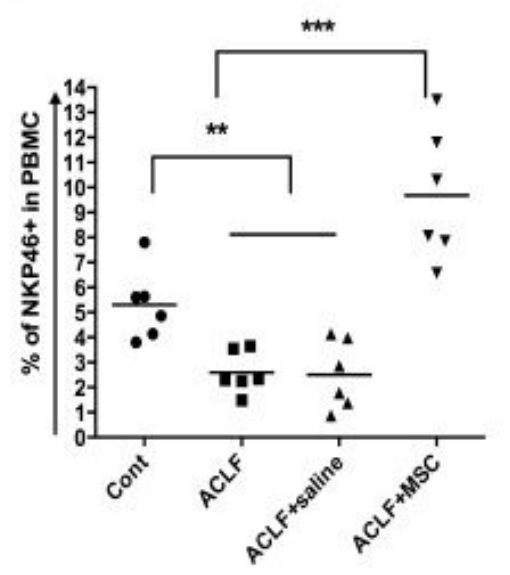

c

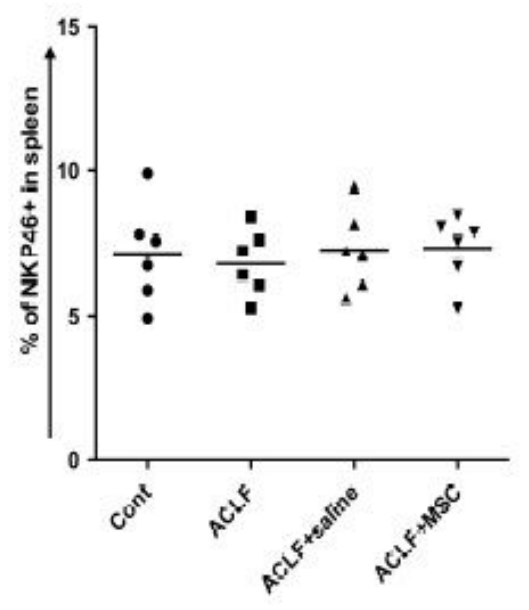

e

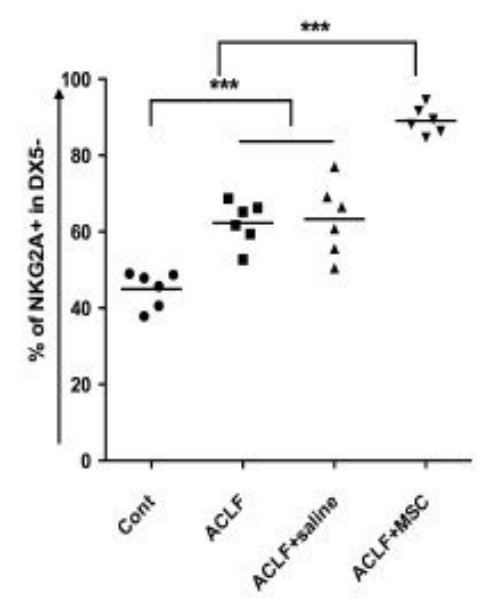

b

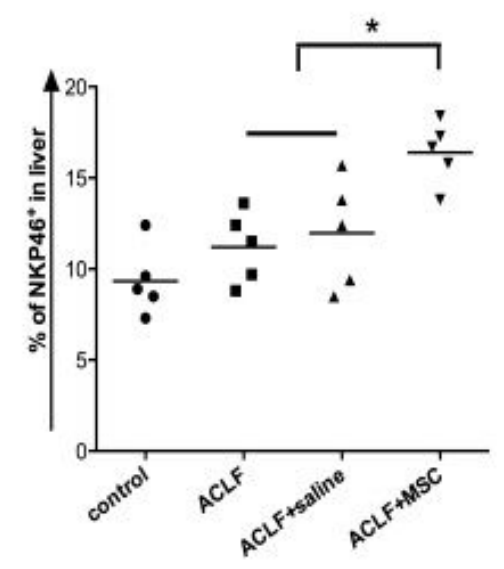

d

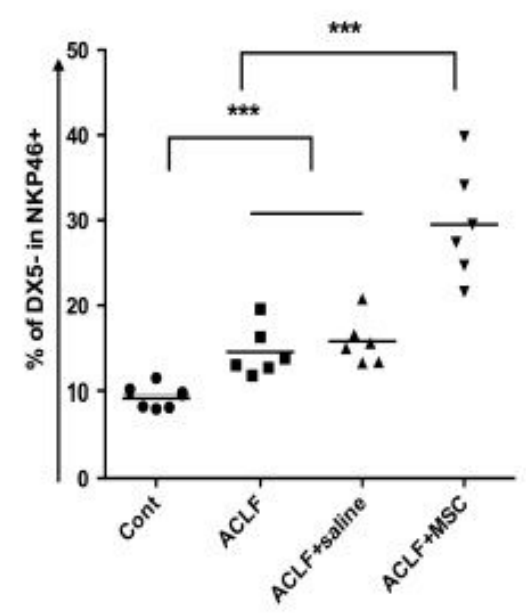

f

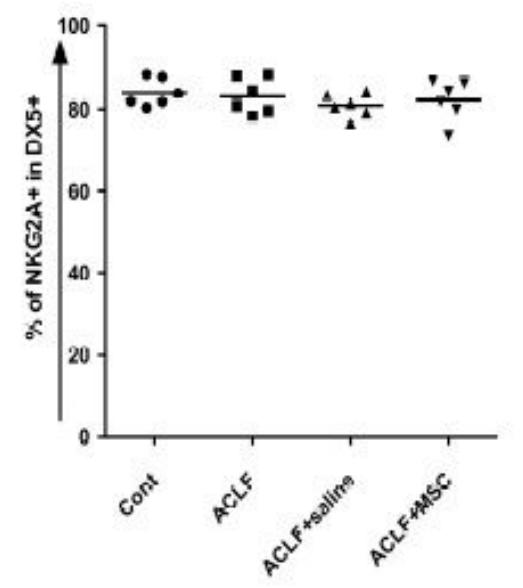

Figure 5

MSC transfusion changed the frequency and phenotype of NKP46+ NK in mice liver. Frequency of NKP46+ cells from total leukocytes from PBMC (a), liver (b) and spleen (c) w/o MSCs transplantation (ACLF+saline and ACLF+MSC) for 48 hours comparing to normal (Cont) and ACLF mice without treatment (ACLF). Student's T test, $n=6$, * indicate $P<0.05$, ${ }^{\star *}$ indicate $P<0.01$, $* \star \star$ indicate $P<0.001$. (d) the frequency of DX5- cells in NKP46+ liver leucocytes, w/o MSCs transplantation (ACLF+saline and 
ACLF+MSC) for 48 hours comparing to normal (Cont) and ACLF mice without treatment (ACLF). Student's $T$ test. $* \star$ indicate $P<0.01$, $* \star *$ indicate $P<0.001$. (e-f) the NKG2A expression frequency on NKP46+DX5- (e) and NKP46+DX5+ (f) liver cells from different groups as A , Student's T test, $n=6$, ** indicate $\mathrm{P}<0.01$, *** indicate $\mathrm{P}<0.001$.

a

NKP46+ blood NK

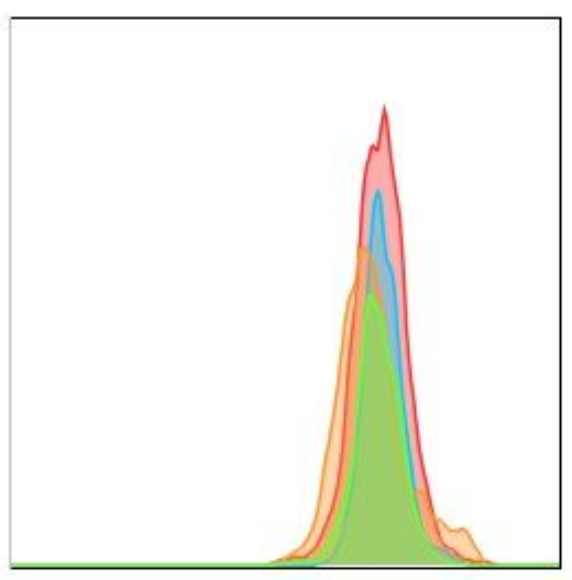

NKP46+DX5+ liver NK

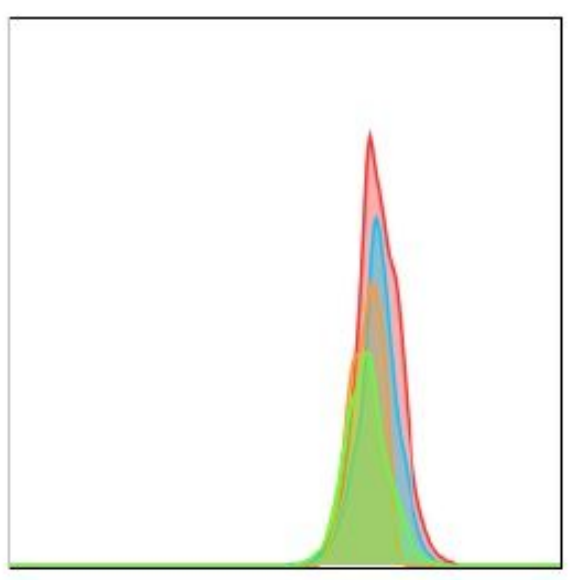

NKP46+DX5- liver NK

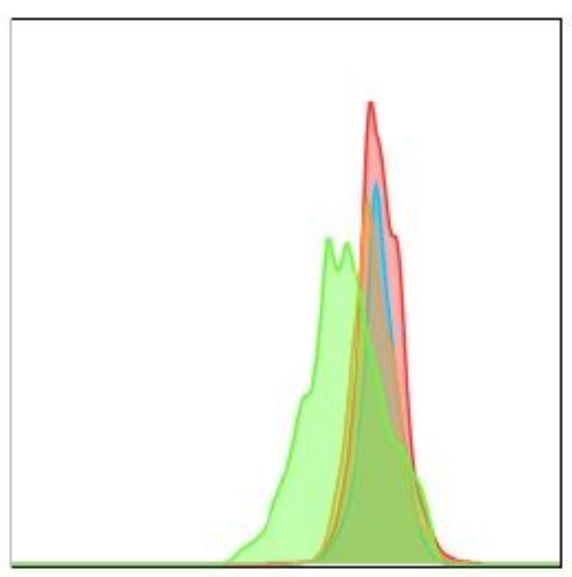

b

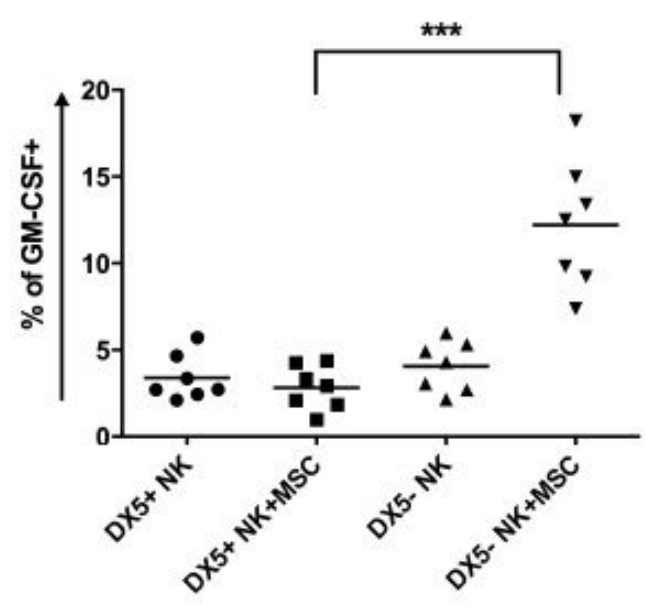

Figure 6

MSCs enhanced DX5- liver NK cells proliferation and GM-CSF production In vitro. (a) Sorting purified NKP46+ blood and liver NKP46+DX5+/- NK cells from normal mice co-culture with/without MSCs (in blue and pink), and NK cells from ACLF mice with/without MSCs (in green and orange) were assessed by proliferation dye after incubation for 48 hours. Overlaid histogram showed from one representative from 3 experiments. (b) Intracellular GM-CSF staining in DX5 positive and negative liver NK cells w/o MSC treatment. GM-CSF secretion increase from DX5- NK cell induced by MSCs . Student's T test, $n=7$, $p<0.0001$. 
a

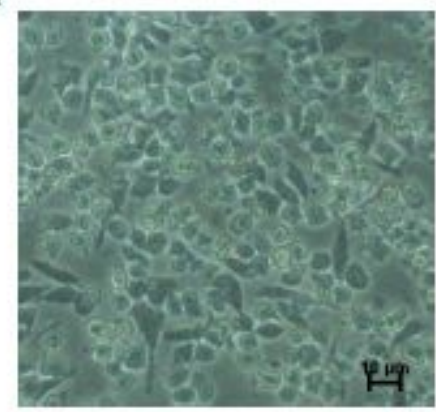

NKP46+DX5+

c

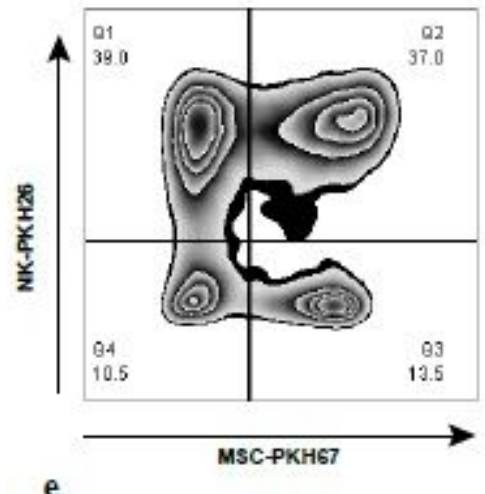

b

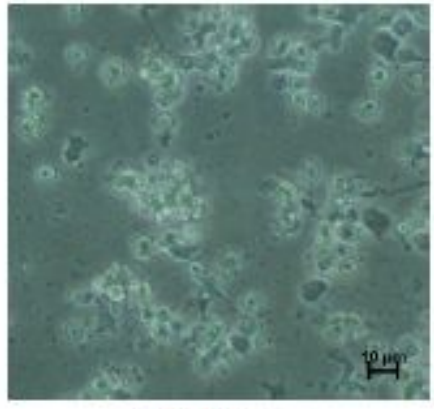

NKP46+DX5-

d

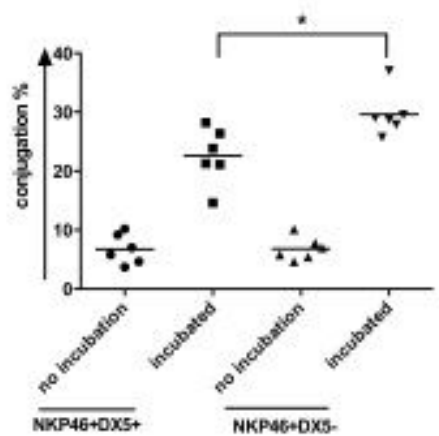

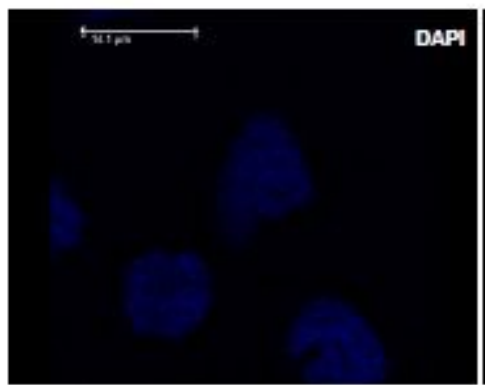
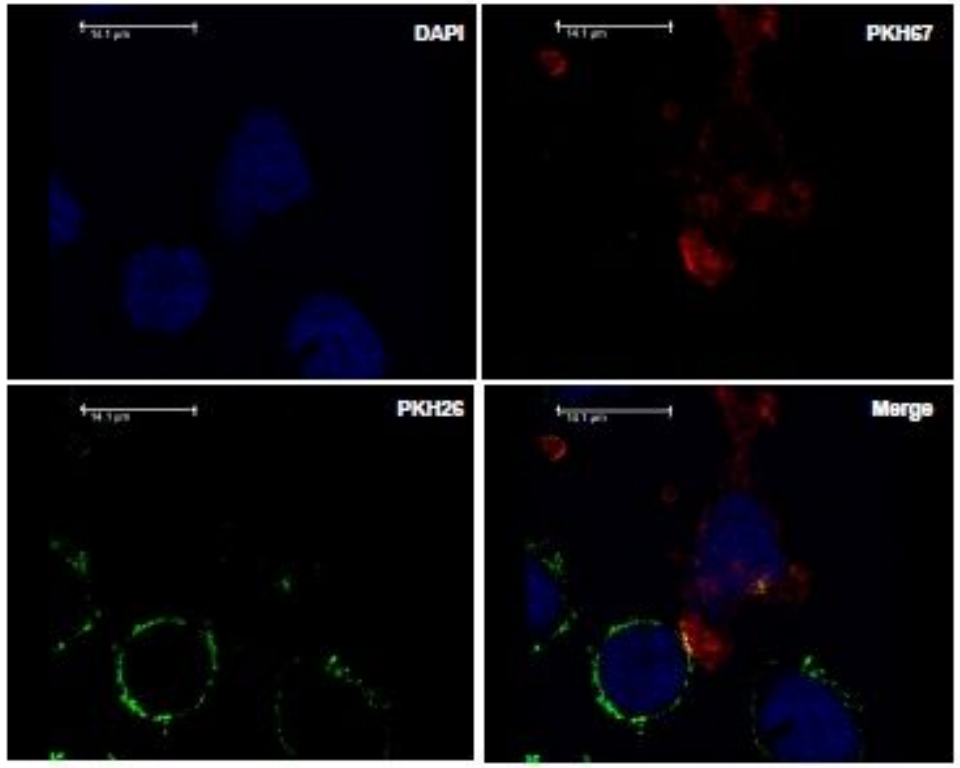

\section{Figure 7}

.MSCs conjugated with NKP46+DX5- liver NK cells preferentially in vitro. Sorting purified NKP46+DX5+ (a) and NKP46+DX5- (b) liver NK cells from ACLF mice co-cultured with pre-coated MSCs in vitro under $100 \mathrm{U} / \mathrm{ml} \mathrm{IL-2}$ for 48 hours, the cells were observed through phase-contrast inverted microscopy. Above images are under 20x amplification. (control NK cells without MSC didn't show for clarification). One typical result showed from 3 experiments with similar results. (c) Sorting purified NKP46+ DX5+/- liver leukocytes cells from ACLF mice were stained with PKH26, co-cultured with/without PKH67 stained MSCs cell. Representative FACS plot to show NK cells conjugated with MSCs in vitro (PKH26/PKH76 
double positive means conjugated cells); (d) conjugation \% was assessed via double positive cells, $n=6$, Student's $T$ test, $P=0.0185$. (e) cell sorter purified NKP46+DX5+ and NKP46+DX5- liver NK cells from ACLF mice were stained by PKH26, added into chamber slides pre-coated with MSCs cells in PKH67, observed by Leica confocal microscope. Above image was from NKP46+DX5- liver NK cells. NKP46+DX5+ which showed no immune synapse with MSCs. Data didn't show for clarification. Experiments repeated 3 times, similar results observed.

\section{Supplementary Files}

This is a list of supplementary files associated with this preprint. Click to download.

- SupplementariesTablesandfigures.doc 